\title{
Utility of a lighted stent to avoid male urethral injury in transanal rectal surgery
}

\author{
Kenji Kawada, Tomoaki Okada, Yoshiharu Sakai \\ Department of Surgery, Graduate School of Medicine, Kyoto University, Kyoto 6068507, Japan.
}

Correspondence to: Dr. Kenji Kawada, Department of Surgery, Kyoto University Graduate School of Medicine, 54 Shogoin Kawahara-cho, Sakyo-ku, Kyoto 6068507, Japan. E-mail: kkawada@kuhp.kyoto-u.ac.jp

How to cite this article: Kawada K, Okada T, Sakai Y. Utility of a lighted stent to avoid male urethral injury in transanal rectal surgery. Mini-invasive Surg 2020;4:7. http://dx.doi.org/10.20517/2574-1225.2019.44

Received: 18 Oct 2019 First Decision: 30 Dec 2019 Revised: 31 Dec 2019 Accepted: 15 Jan 2020 Published: 12 Feb 2020

Science Editor: Giulio Belli Copy Editor: Jing-Wen Zhang Production Editor: Tian Zhang

\begin{abstract}
Total mesorectal excision (TME) is accepted as the standard technique in rectal surgery. In recent years, significant attention has been focused on transanal TME (taTME) as a promising approach for rectal cancer. However, this approach can involve an inherent risk of male urethral injury, because there is no clear anatomical border between the rectal muscularis propria and rectourethral muscle. We used a lighted urethral stent to identify the urethra during taTME for 6 patients with distal rectal cancer. In five of six cases, an infrared-detecting camera could detect a red fluorescent signal from the lighted urethral stent during the anterior dissection of the rectum, which helped us to determine the correct dissection line. A lighted urethral stent is a useful tool that helps visualize the urethra during taTME and improves taTME applicability in clinical practice.
\end{abstract}

Keywords: Transanal total mesorectal excision, lighted urethral stent, urethral injury

\section{INTRODUCTION}

Total mesorectal excision (TME), introduced by Heald, is accepted as the standard technique in rectal surgery $^{[1]}$. As compared to open surgery, laparoscopic surgery can provide better visibility in a narrow pelvic space, which enables surgeons to conduct precise TME surgery. Laparoscopic rectal surgery has been accepted based on accumulating evidence; however, it remains technically difficult in cases with obesity and/or a narrow pelvis. Recent randomized controlled trials (RCTs) (i.e., COLOR II and COREAN) exhibited more favorable outcomes of laparoscopic rectal surgery compared with open rectal surgery ${ }^{[2,3]}$, whereas other recent RCTs (i.e., ALaCaRT and ACOSOG Z6051) did not ${ }^{[4,5]}$. In recent years, transanal TME (taTME) surgery for rectal cancer has attracted intense attention due to the improvements in the

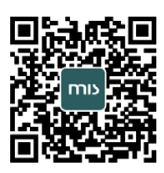




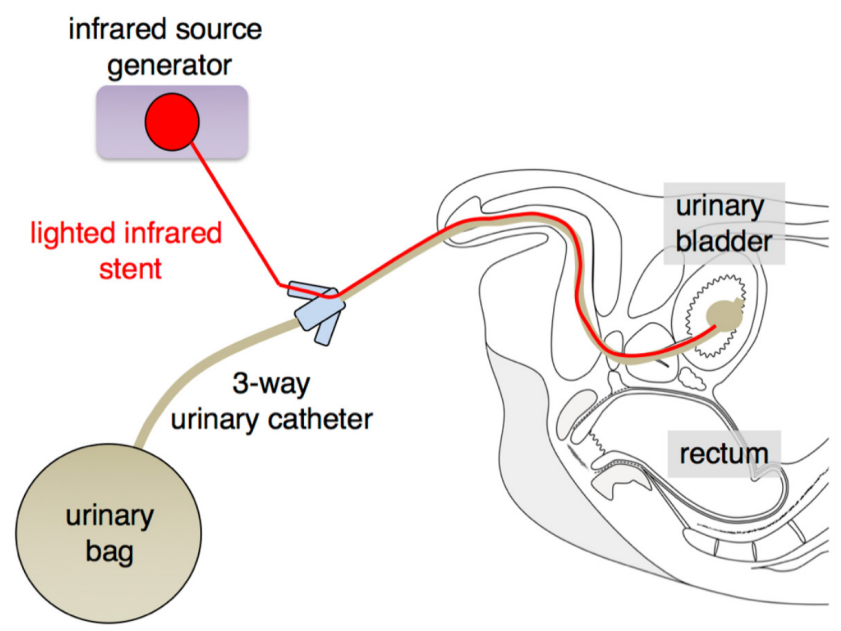

Figure 1. Setup of a lighted stent

quality of TME. However, this approach can involve an inherent risk of male urethral injury during anterior dissection of the rectum ${ }^{[6-10]}$, because there is no clear anatomical border between the rectal muscularis propria and rectourethral muscle. In taTME surgery, anatomical knowledge of the male anterior anorectum is critical to avoid male urethral injury and rectal perforation. We recently reported the three-dimensional morphology of the male anterior anorectum based on the histological analyses of male cadavers ${ }^{[11]}$. In clinical practice, limited studies have reported on the utility of a lighted urethral stent during taTME surgery ${ }^{[6,7,10]}$. In this study, we show the anatomical findings of the anterior anorectum in a cadaveric study as well as the availability of a lighted urethral stent in a clinical setting.

\section{METHODS}

In a cadaveric study, gelatin-embedded male pelvises were sectioned; the specimens including the anterior anorectum were subsequently dissected for histological examination, as described previously ${ }^{[11]}$. Paraffin-embedded serial sections at $10 \mathrm{~m}$ were used for Elastica van Gieson (EVG) staining and immunohistochemical analysis with antibodies against smooth muscle actin (Smooth Muscle Ab-1, Thermo Fisher Scientific) and skeletal muscle myosin (Skeletal Muscle Ab-2, Thermo Fisher Scientific) ${ }^{[11]}$. This study was conducted following the Act on Body Donation for Medical \& Dental Education law of Japan.

In a clinical study, we used a lighted stent (Infrared Illumination System, Stryker. Inc.) to identify the urethra during taTME in six patients with distal rectal cancer. For visualization of the urethra, a lighted stent was preoperatively introduced into a three-way urinary catheter (\#18Fr Foley), which was placed into the bladder [Figure 1]. The lead of a lighted stent was connected to an external infrared source generator. The wavelength of the lighted stent was approximately $830 \mathrm{~nm}$, and, hence, an infrared-detecting camera system (1588 AIM Platform, Stryker) was employed to detect a fluorescent signal from the lighted stent. Informed consent was obtained from all patients. The study protocols were approved by the Institutional Review Board of Kyoto University.

\section{RESULTS}

Figure 2 shows histological sections of the anterior anorectum in the cadaveric study. The urethra was very close to the rectal muscularis propria just inferior to the apex of prostate. In the horizontal section, striated muscle fibers of the puborectalis muscle surrounded the rectal muscularis propria from the anterolateral side to the posterior side. Abundant smooth muscle containing collagen fibers (i.e., rectourethral muscle) extended anteriorly from the longitudinal muscle of the rectal muscularis propria [Figure $2 \mathrm{~A}$ ]. In the 


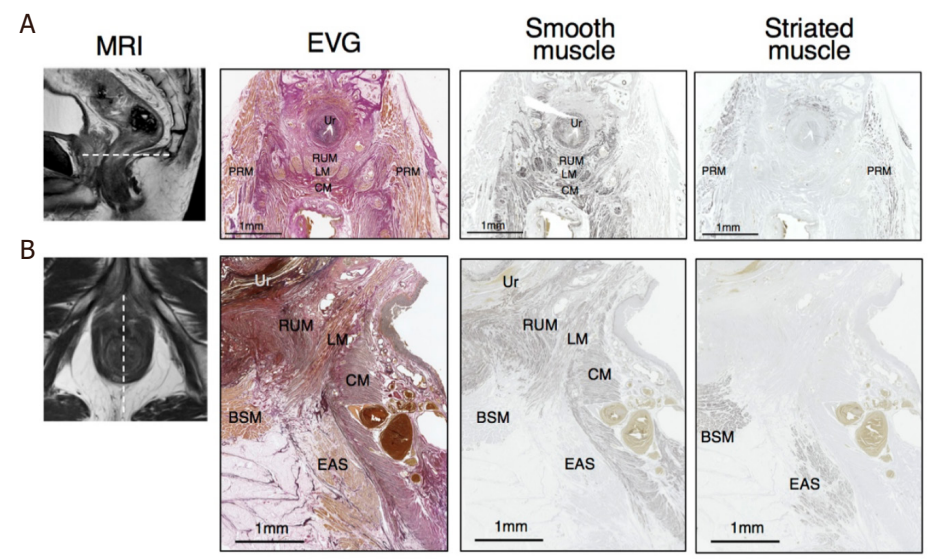

Figure 2. Histological sections. EVG, anti-smooth muscle, and anti-striated muscle staining: (A) horizontal sections; and (B) Sagittal sections. BSM: bulbospongiosus muscle; CM: circular muscle layer; EAS: external anal sphincter; LM: longitudinal muscle layer; PRM: puborectalis muscle; RUM: rectourethral muscle; Ur: urethra; EVG: elastica van gieson; MRI: magnetic resonance imaging

Normal mode

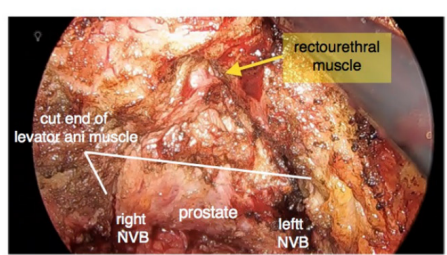

Turned-off mode

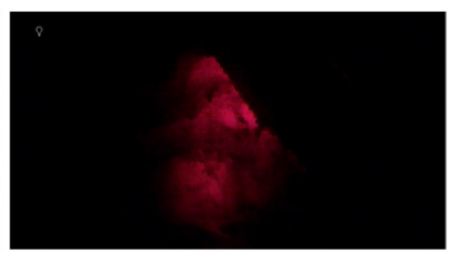

Figure 3. Transanal view of the anterior anorectum in Case 2. Normal mode (left) and turned-off mode (right) are shown. The rectourethral muscle, NVB, and cut end of the levator ani muscle are shown. NVB: neurovascular bundle

sagittal section, the rectourethral muscle occupied the posteroinferior area of the urethra from the upper border of the external anal sphincter muscle to the apex of the prostate [Figure $2 \mathrm{~B}$ ]. There was no clear anatomical border between the rectourethral muscle and longitudinal muscle, indicating that surgeons need to pay attention to urethral injury during division of the rectourethral muscle in taTME.

We used a lighted stent to identify the urethra in six taTME surgeries: abdominoperineal resection (APR; $n=4$ ) and intersphincteric resection (ISR; $n=2$ ). Vessel ligations and mobilization of the left-sided colon were laparoscopically performed. The perineal approach was conducted under direct vision to attach a GelPoint Mini port device (Applied Medical). After GelPoint Mini was placed, taTME was initiated. The posterior side of the rectum was first dissected until the sacral promontory was reached. Next, the dissection was extended toward the lateral side. Bilateral pelvic splanchnic nerves were preserved at the 5 and 7 o'clock positions. On the anterior side, the correct dissection line could not be easily identified because there was no clear anatomical border. Therefore, an infrared-detecting camera (1588 AIM) was used to detect a red fluorescent signal from a lighted urethral stent in real time during the anterior dissection of the rectum. Figure 3 shows the views of the anterior side in Case 2 (APR). The red fluorescent signal could be detected during division of the rectourethral muscle. The signal was bright with low background under the turned-off mode. Anterior dissection was conducted using fluorescent information as reference. The red-lighted area was located between the superior part of membranous urethra and the inferior lobe of prostate. In five of six cases (i.e., Cases 1-4 and 6), we could detect the red fluorescent signal from a lighted urethral stent under the turned-off mode [Figure 4]. The portion of the lighted area was similar in the five cases, although the fluorescence intensity was slightly different depending on the angle of the lighted stent. Under the normal light mode, the fluorescent signal was not detected in all six cases. In 


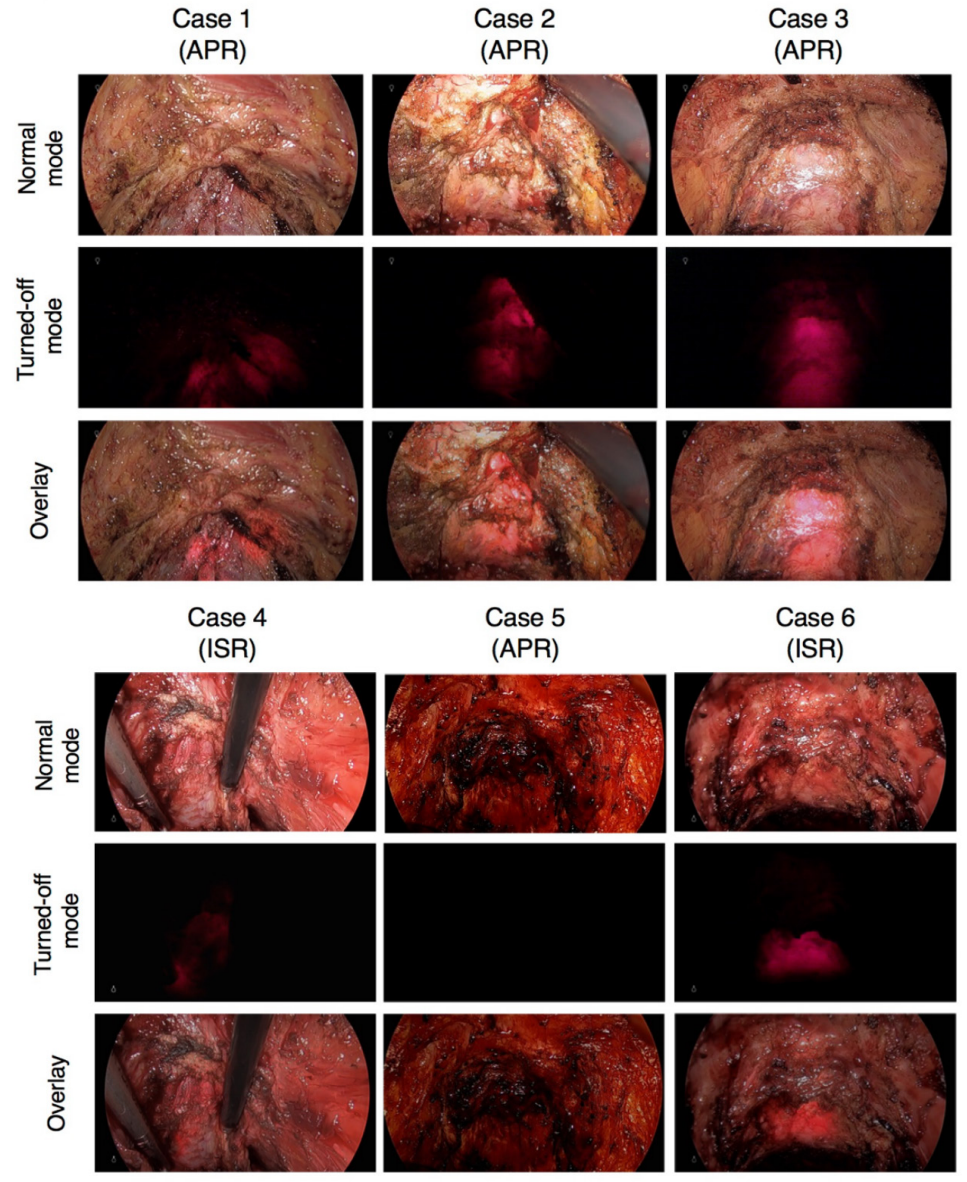

Figure 4. Transanal view of the anterior anorectum in six cases. Normal mode (top), turned-off mode (middle), and overlaid view (bottom) are shown. APR: abdominoperineal resection; ISR: intersphincteric resection

Case 4, the fluorescent signal was weak under the turned-off mode. When the prostate was broadly pressed by the forceps, the fluorescent signal was slightly enhanced. In Case 5, we could not detect a fluorescent signal even if the prostate was broadly pressed. No significant complications were observed in all cases.

\section{DISCUSSION}

We previously reported a cadaveric study about the visualization of a lighted urethral stent during transanal ISR ${ }^{[9]}$. In the correct dissection plane that resulted in preservation of the urethra, a fluorescent signal was barely identified under the normal light mode, while it could be clearly detected under the turned-off mode. In the incorrect (i.e., deeper) dissection plane that resulted in urethral injury, the lighted urethral stent was clearly detected under both the normal light mode and turned-off mode. Identification of the urethra using the lighted urethral stent under the turned-off mode could be helpful to avoid inadvertent urethral injury during the anterior dissection of the rectum.

In the present study, a fluorescent signal from a lighted urethral stent could not be detected in one case (Case 5). It is not clear why a fluorescent signal could not be detected. Although the lighted stent was designed to illuminate through up to $12 \mathrm{~mm}$ tissues, the intensity of the fluorescent signal can be affected by some factors: for example, the thickness of the tissues covering the urethra and prostate, the distance between the lighted stent and laparoscopic camera, the rotation angle of the urinary catheter, and the equipment condition. Further investigation is needed to validate our findings. 


\section{CONCLUSION}

A lighted urethral stent is useful to visualize the urethra during taTME surgery in clinical practice. The assistance of fluorescent information helped in reducing the risk of urethral injury on the anterior side.

\section{DECLARATIONS}

\section{Authors' contributions}

Conception and study design: Kawada K, Sakai Y

Data acquisition and interpretation: Kawada K, Okada T

Drafting of manuscript: Kawada K

Critical revision for intellectual content: Okada T, Sakai Y

\section{Availability of data and materials}

Not applicable.

\section{Financial support and sponsorship}

This study was supported by the Japan Society of Laparoscopic Colorectal Surgery.

\section{Conflicts of interest}

All authors declared that there are no conflicts of interest.

\section{Ethical approval and consent to participate}

A cadaveric study was conducted following the Act on Body Donation for Medical \& Dental Education law of Japan. In a clinical study, informed consent was preoperatively obtained from all patients. This study was conducted in accordance with the ethical principal that have their origins in the Declaration Helsinki, and the protocols were approved by the Institutional Review Board of Kyoto University.

\section{Consent for publication}

Not applicable.

\section{Copyright}

(c) The Author(s) 2020.

\section{REFERENCES}

1. Heald RJ, Husband EM, Ryall RD. The mesorectum in rectal cancer surgery--the clue to pelvic recurrence? Br J Surg 1982;69:613-6.

2. Bonjer HJ, Deijen CL, Abis GA, Cuesta MA, van der Pas MH, et al. A randomized trial of laparoscopic versus open surgery for rectal cancer. N Engl J Med 2015;372:1324-32.

3. Jeong SY, Park JW, Nam BH, Kim S, Kang SB, et al. Open versus laparoscopic surgery for mid-rectal or low-rectal cancer after neoadjuvant chemoradiotherapy (COREAN trial): survival outcomes of an open-label, non-inferiority, randomised controlled trial. Lancet Oncol 2014;15:767-74.

4. Fleshman J, Branda M, Sargent DJ, Boller AM, George V, et al. Effect of laparoscopic-assisted resection vs open resection of stage ii or iii rectal cancer on pathologic outcomes: the ACOSOG Z6051 randomized clinical trial. JAMA 2015;314:1346-55.

5. Stevenson AR, Solomon MJ, Lumley JW, Hewett P, Clouston AD, et al. Effect of laparoscopic-assisted resection vs open resection on pathological outcomes in rectal cancer: the ALaCaRT randomized clinical trial. JAMA 2015;314:1356-63.

6. Atallah S, Martin-Perez B, Drake J, Stotland P, Ashamalla S, et al. The use of a lighted stent as a method for identifying the urethra in male patients undergoing transanal total mesorectal excision: a video demonstration. Tech Coloproctol 2015;19;6:375

7. Mabardy A, Lee L, Valpato AP, Atallah S. Transanal total mesorectal excision with intersphincteric resection and use of fluorescent angiography and a lighted urethral stent for distal rectal cancer. Tech Coloproctol 2017;21:581-2.

8. Barnes TG, Penna M, Hompes R, Cunningham C. Fluorescence to highlight the urethra: a human cadaveric study. Tech Coloproctol 2017;21:439-44.

9. Okada T, Kawada K, Nakamura T, Okamura R, Hida K, et al. A cadaveric demonstration of visualization of the urethra using a lighted stent during transanal intersphincteric resection. Int Cancer Conf J 2018;7:77-80.

10. Kawada K, Hida K, Yoshitomi M, Sakai Y. Use of a lighted stent to identify the urethra during transanal abdominoperineal resection. 
Tech Coloproctol 2018;22:467-8.

11. Okada T, Hasegawa S, Nakamura T, Hida K, Kawada K, et al. Precise three-dimensional morphology of the male anterior anorectum reconstructed from large serial histological sections: a cadaveric study. Dis Colon Rectum 2019;62:1238-47. 